\title{
Condición de los arrecifes coralinos del Parque Nacional Guanahacabibes, Cuba
}

\section{Condition of coral reefs in the Guanahacabibes National Park, Cuba}

\author{
Susana Perera Valderrama ${ }^{1 *}$, Pedro M. Alcolado ${ }^{2}$, Hansel Caballero Aragón ${ }^{3}$, Elena de la \\ Guardia Llansó ${ }^{4}$ y Dorka Cobián Rojas ${ }^{5}$
}

\section{RESUMEN}

En el 2007 se evaluó el estado de salud de los arrecifes coralinos del Parque Nacional Guanahacabibes, Cuba, sobre la base de la condición de las comunidades de corales. Se analizaron indicadores de condición de los corales escleractinios (diámetro máximo, riqueza de especies, enfermedades y mortalidad) a distintas profundidades, en 13 estaciones con diferentes niveles de exposición a los principales fenómenos océano-atmosféricos generadores de oleaje en el área (vientos nortes, sures y alisios). Se identificaron 42 especies de corales pétreos. Las más abundantes fueron Siderastrea siderea, Montastraea faveolata, Agaricia agaricites y Porites astreoides. El promedio de cobertura de coral vivo varió entre $4.8 \%$ y $31.2 \%$, con un promedio general de $17 \%$. El análisis integrado de todos los indicadores de condición de las comunidades de corales sugirió la existencia de dos áreas diferenciadas según la exposición a los vientos y el consecuente efecto del oleaje y los sedimentos. La primera área abarcó el este de la Bahía de Corrientes (entre Uvero Quemado y Cuevas de Pedro), la más resguardada, y donde los indicadores biológicos sugieren un ambiente más favorable. La segunda comprendió la parte occidental más expuesta (entre Veral y Faro Roncali), que presentó la mayor cobertura relativa del conjunto de especies resistentes a la sedimentación, especialmente en 10 y $15 \mathrm{~m}$ de profundidad. Estos resultados indican que los arrecifes del Parque Nacional Guanahacabibes no escapan del proceso de degradación que afrontan la mayoría de los arrecifes de la región del Caribe.

Palabras claves: Corales pétreos, arrecifes coralinos, Cuba.

\begin{abstract}
In 2007, a health assessment of coral reefs in the Guanahacabibes National Park, Cuba, was conducted based on the condition of coral communities. Coral condition indicators (maximum diameter, species richness, diseases and mortality) were evaluated, at different depths in 13 sites with varying exposure levels to the main ocean-atmospheric phenomena that generate waves in the area (north, south and trade winds). Forty two species of stony corals were identified. The most abundant species were Siderastrea siderea, Montastraea faveolata, Agaricia agaricites and Porites astreoides. The average coral cover varied between $4.8 \%$ and $31.2 \%$, with a general average of $17 \%$. The integrated analysis of all condition indicators of coral communities suggested the existence of two distinctive areas according to wind exposure and the consequent effect of both waves and sediments. The first area, covering east of Bahía de Corrientes (between Uvero Quemado and Cuevas de Pedro), was more protected and had a more favorable environment, as suggested by the biological indicators. The second area was comprised of the western part, was more exposed (between Veral and Roncali), and showed the highest relative cover of species resistant to sedimentation, especially at 10 and $15 \mathrm{~m}$ deep. These results indicate that coral reefs at the Guanahacabibes National Park do not escape from the degradation process faced by most coral reefs in the Caribbean region.
\end{abstract}

Keywords: Stony corals, coral reefs, Cuba.

\footnotetext{
1 Centro Nacional de Áreas Protegidas. CITMA. $18^{\text {a }}$ No. 4114. Playa. La Habana. Cuba. susana@snap.cu* Instituto de Oceanología. CITMA. Ave. 1ra. No. 18406, entre 184 y 186, Flores, Playa, Ciudad de la Habana, Cuba Acuario Nacional de Cuba. CITMA. Acuario Nacional, Avenida 1ra y 60, Playa, La Habana, Cuba Empresa Nacional para la Protección de la Flora y la Fauna. MINAG. Ave Rancho Boyeros Boyeros, La Habana. Cuba Parque Nacional Guanahacabibes. CITMA. La Bajada. Pinar del Río
}

Recibido 26 II 2013

Aceptado 23 VII 2013

DOI: http://dx.doi.org/10.15359/revmar.5.5 


\section{INTRODUCCIÓN}

Los arrecifes coralinos constituyen uno de los ecosistemas más diversos del mundo, habitados por cerca del $25 \%$ de todas las especies marinas (Töpfer, 2003). Estos poseen una importancia vital asociada a los bienes y servicios que brindan a las naciones costeras tropicales y subtropicales que dependen en gran medida de sus recursos (Hoegh-Guldberg, 2004).

Se conoce que factores físicos como la profundidad, la luz, el ángulo de disposición de las colonias inciden en la estructura de los arrecifes y en los esquemas de abundancia y distribución de las especies de coral que lo forman (Díaz-Pulido et al. 2004). Estas condiciones aportan un determinado nivel de resiliencia a los arrecifes e inciden en su capacidad de supervivencia y recuperación ante las principales amenazas que le impone el mundo moderno: la sobrepesca, el desarrollo costero y el cambio climático global (Lesser et al. 2007). Según Torres \& Morelock (2002), en la capacidad de supervivencia de los corales pétreos está implicada en gran medida, además, la resistencia al oleaje y el efecto de los sedimentos. En ese sentido, los autores destacan como especies resistentes a la sedimentación y al oleaje a Siderastrea siderea, Porites astreoides, Diploria strigosa y Montastraea cavernosa, de lo que puede deducirse el potencial del predominio de estas especies como indicador de la intensidad de estos factores.
Los arrecifes costeros de la Península de Guanahacabibes poseen aproximadamente $80 \mathrm{~km}$ de extensión y están incluidos en el Parque Nacional Guanahacabibes, que fue declarado legalmente en el 2001. Desde ese mismo año, esta área fue declarada por el Ministerio de la Pesca como Zona Bajo Régimen Especial de Uso Protección, donde se prohibió la pesca comercial, submarina y deportivarecreativa (Cuba, 2001a). Hasta el presente, el uso de la zona marina del parque ha estado restringido fundamentalmente al buceo recreativo con fines turísticos en arrecifes de coral en el extremo este de la península.

Las comunidades de corales pétreos del Parque Nacional Guanahacabibes han sido poco estudiadas, y en su mayoría, los trabajos realizados (Alcolado et al. 2003; Caballero et al. 2007 y González-Ferrer et al. 2007) se han enfocado en la descripción del estado de salud de los puntos de buceo del Centro Internacional María la Gorda, al este de la Bahía de Corrientes. Los arrecifes de los puntos de buceo de María la Gorda, calificados por estos autores como de excelente desarrollo estructural y de muy buen estado de conservación, solo representan el 15\% de los arrecifes del área protegida. Por ese motivo se desconoce el estado de salud de las comunidades de corales en otras zonas del parque.

De esta forma, la zona arrecifal de la península se puede considerar propicia para estudiar la influencia de los eventos generadores de oleaje y desestabilizadores de sedimentos (vientos nortes, sures $\mathrm{y}$ alisios) sobre las comunidades de corales, pues la configuración geográfica 
de la línea de costa ofrece diferentes niveles de exposición de los arrecifes. Además, por el bajo nivel de impacto humano están minimizados los efectos antrópicos (contaminación, pesca comercial y turismo masivo) sobre estas comunidades, en la interpretación de la influencia de los factores naturales de interés. Por otra parte, el manejo de los ecosistemas y la implementación del área marina protegida requieren una comprensión adecuada de los procesos de mantenimiento de la diversidad, así como un entendimiento adecuado de las escalas a las que estos procesos operan (Zvuloni et al. 2010).

Por estas razones, este trabajo está dirigido a evaluar el estado de salud de las comunidades de corales pétreos de los arrecifes del Parque Nacional Guanahacabibes y a analizar cómo las diferentes condiciones hidrodinámicas generadas por los vientos y el grado de exposición pueden haber influido en su composición, estructura y salud.

\section{MATERIALES Y MÉTODOS}

El Parque Nacional Guanahacabibes constituye la zona núcleo más importante de la Reserva de Biosfera Península de Guanahacabibes, provincia Pinar del Río. Incluye 39830 ha, de las cuales 15950 ha son marinas y se extienden por toda la costa sur de la península, desde Jaimanitas al este de Cabo Corrientes hasta el Cabo de San Antonio (Fig. 1) (Cuba, 2001b).

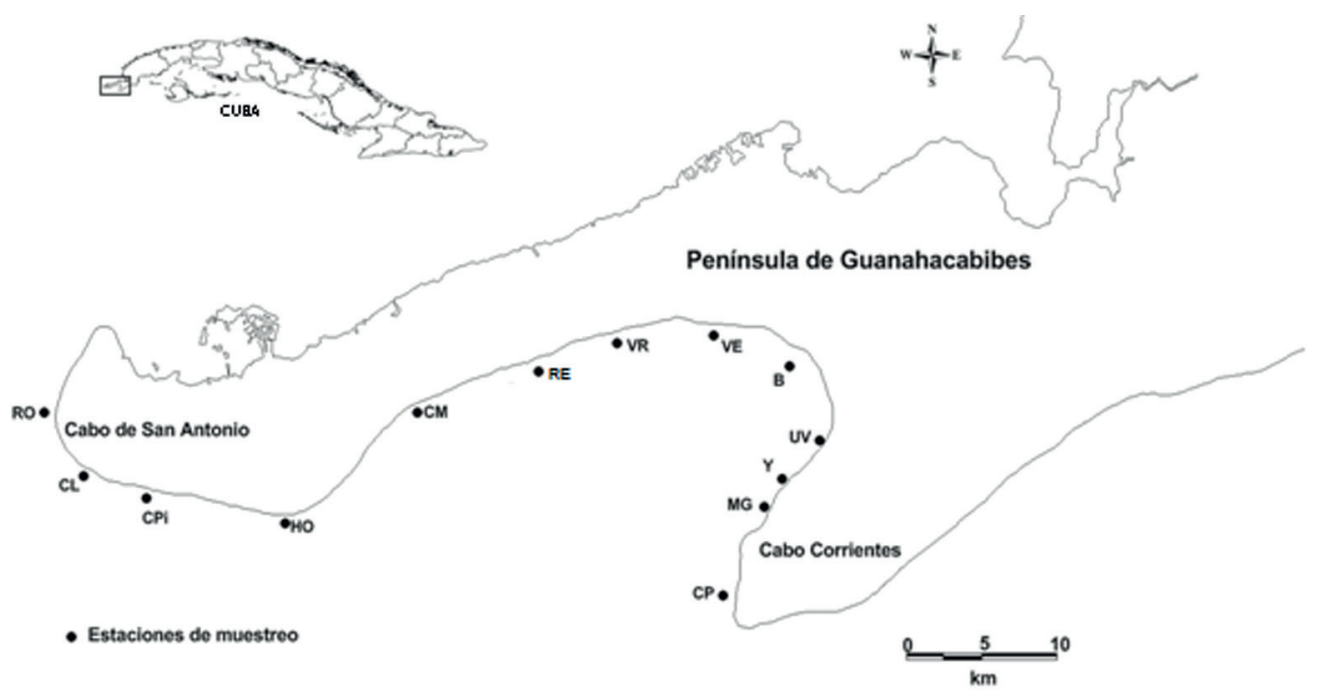

Fig. 1. Ubicación geográfica del Parque Nacional Guanahacabibes y de las estaciones muestreadas. RO: Faro Roncali, CL: Caleta Larga, CPi: Caleta del Piojo, HO: El Holandés, CM: Caleta del Mangle, RE: Resguardo, VR: Verraco, VE: Veral, B: La Bajada, UV: Uvero, Y: Yemayá, MG: María la Gorda, CP: Cuevas de Pedro

Fig. 1. Location of the Guanahacabibes National Park and the studied sites. RO: Faro Roncali, CL: Caleta Larga, CPi: Caleta del Piojo, HO: El Holandés, CM: Caleta del Mangle, RE: Resguardo, VR: Verraco, VE: Veral, B: La Bajada, UV: Uvero, Y: Yemayá, MG: María la Gorda, CP: Cuevas de Pedro 
La zona costera del Parque Nacional Guanahacabibes es predominantemente cárstica con segmentos de playa, por lo que la red hidráulica natural es muy limitada y son escasas las descargas al mar (Iturralde-Vinent, 2004). De noviembre a abril predominan vientos del primer cuadrante, debido a la influencia de los sistemas de la temporada invernal (Rodríguez et al. 1984), mientras que en el verano los rumbos giran más al sudeste (Rodríguez-Ramírez \& Ballester, 1991). La presencia de las zonas de bajas presiones en el Golfo de México en los meses de febrero y marzo, la proximidad y avance de los sistemas frontales y la profundidad de la dorsal anticiclónica en verano hacia el Caribe son factores que determinan la ocurrencia de vientos de la región sur en el área (Sánchez-Díaz \& Victorero-Hernández, 2009).

En el Parque Nacional Guanahacabibes, los arrecifes son predominantemente de borde de plataforma o costeros, con perfil de terraza única que termina en un escarpe profundo, $\mathrm{y}$ en algunas estaciones se presenta un escarpe somero o primer veril que divide a la terraza en una somera y otra profunda (González et al. 2007).

Para caracterizar las comunidades de corales pétreos y determinar la influencia de la exposición a los regímenes de vientos más fuertes, a través de los efectos de la acción conjunta del oleaje y de los sedimentos generados por aquellos, sobre sus indicadores de condición, se realizó una expedición de muestreo en febrero de 2007. Se establecieron 13 estaciones de muestreo más o menos equidistantes a lo largo de la costa sur y sudeste de la Península de Guanahacabibes (Fig. 1). En cada estación se trazó un perfil perpendicular a la línea de costa sobre el que se ubicaron estaciones a $5,10,15$ y 20 metros de profundidad (Cuadro 1).

Se utilizó el método del transecto lineal de $10 \mathrm{~m}$ como unidad de muestreo, como se describe en el protocolo AGRRA (2000). En cada estación se desplegaron 15 transectos, los cuales se ubicaron de acuerdo con la morfología del arrecife y fueron distribuidos al azar. En cada transecto se estimó la cobertura del fondo por tejido vivo de coral. A cada coral de $10 \mathrm{~cm}$ o más de diámetro, interceptado por el transecto, se le midió la cobertura lineal de coral vivo debajo del transecto, el diámetro máximo, el porcentaje de superficie con mortalidad antigua (partes muertas del coral donde los coralites han desaparecido o han sido cubiertos por organismos incrustantes) y reciente (partes muertas del coral, generalmente blancas e intactas, en las que es posible identificar la especie), y se anotó la presencia de blanqueamiento y otras enfermedades. Para la identificación de los corales duros (órdenes Scleractinia y Capitata) se siguieron los criterios de Steneck et al. (1997).

Se determinó, tanto para el área como para las estaciones, la cobertura de coral vivo general y relativa de cada especie (porcentaje de la cobertura de coral vivo de la especie en relación con el porcentaje total de cobertura de coral vivo). Con este último criterio se 
Cuadro 1. Descripción de las estaciones de muestreo para la caracterización de las comunidades de corales. Con asteriscos se indican las estaciones donde no se realizaron muestreos por la ausencia de desarrollo coralino debido a la no existencia de sustrato adecuado

Table 1. Description of the studied sites for the characterization of the coral communities. The sites where samples were not taken due to absence of coral development for the lack of an adequate substratum are marked with an asterisk

\begin{tabular}{|c|c|c|c|c|c|c|}
\hline \multirow{2}{*}{ Estaciones } & \multicolumn{2}{|c|}{ Coordenadas } & \multicolumn{4}{|c|}{ Profundidades } \\
\hline & Latitud & Longitud & $5 \mathrm{~m}$ & $10 \mathrm{~m}$ & $15 \mathrm{~m}$ & $20 \mathrm{~m}$ \\
\hline $\begin{array}{l}\text { Cuevas de } \\
\text { Pedro }\end{array}$ & 21.7762 & -84.5202 & $\begin{array}{l}\text { Arena y } \\
\text { cascajos* }\end{array}$ & $\begin{array}{l}\text { Camellones y } \\
\text { canales }\end{array}$ & $\begin{array}{l}\text { Cima del } \\
\text { umbral }\end{array}$ & $\begin{array}{l}\text { Borde exterior del } \\
\text { umbral }\end{array}$ \\
\hline $\begin{array}{l}\text { María la } \\
\text { Gorda }\end{array}$ & 21.8124 & -84.5039 & $\begin{array}{l}\text { Cabezos } \\
\text { coralinos }\end{array}$ & Veril & Arenal* & $\begin{array}{l}\text { Borde superior del } \\
\text { umbral }\end{array}$ \\
\hline Yemayá & 21.8345 & -84.4910 & $\begin{array}{l}\text { Cabezos } \\
\text { coralinos }\end{array}$ & Arenal* & $\begin{array}{l}\text { Cima del } \\
\text { umbral }\end{array}$ & $\begin{array}{l}\text { Borde exterior del } \\
\text { umbral }\end{array}$ \\
\hline $\begin{array}{l}\text { Uvero } \\
\text { Quemado }\end{array}$ & 21.8603 & -84.4611 & $\begin{array}{l}\text { Cabezos } \\
\text { coralinos }\end{array}$ & $\begin{array}{l}\text { Cabezos } \\
\text { coralinos }\end{array}$ & $\begin{array}{l}\text { Cima del } \\
\text { umbral }\end{array}$ & $\begin{array}{l}\text { Borde exterior del } \\
\text { umbral }\end{array}$ \\
\hline La Bajada & 21.9204 & -84.4827 & $\begin{array}{l}\text { Camellones y } \\
\text { canales }\end{array}$ & $\begin{array}{l}\text { Camellones y } \\
\text { canales }\end{array}$ & $\begin{array}{l}\text { Camellones y } \\
\text { canales }\end{array}$ & $\begin{array}{l}\text { Camellones y } \\
\text { canales con poca } \\
\text { pendiente }\end{array}$ \\
\hline Veral & 21.9332 & -84.5455 & $\begin{array}{l}\text { Terraza } \\
\text { rocosa }\end{array}$ & Arenal* & $\begin{array}{l}\text { Camellones y } \\
\text { canales }\end{array}$ & $\begin{array}{l}\text { Borde exterior del } \\
\text { umbral }\end{array}$ \\
\hline Verraco & 21.9197 & -84.6149 & $\begin{array}{l}\text { Terraza } \\
\text { rocosa }\end{array}$ & $\begin{array}{l}\text { Camellones y } \\
\text { canales }\end{array}$ & $\begin{array}{l}\text { Camellones y } \\
\text { canales }\end{array}$ & $\begin{array}{l}\text { Camellones y } \\
\text { canales con poca } \\
\text { pendiente }\end{array}$ \\
\hline Resguardo & 21.8964 & -84.6829 & $\begin{array}{l}\text { Terraza } \\
\text { areno-rocosa }\end{array}$ & Arenal* & $\begin{array}{l}\text { Camellones y } \\
\text { canales }\end{array}$ & $\begin{array}{l}\text { Camellones } \\
\text { y canales con } \\
\text { pendiente abrupta }\end{array}$ \\
\hline $\begin{array}{l}\text { Caleta del } \\
\text { Mangle }\end{array}$ & 21.8619 & -84.7460 & $\begin{array}{l}\text { Terraza } \\
\text { rocosa }\end{array}$ & $\begin{array}{l}\text { Camellones y } \\
\text { canales }\end{array}$ & $\begin{array}{l}\text { Camellones y } \\
\text { canales }\end{array}$ & $\begin{array}{l}\text { Camellones y } \\
\text { canales cayendo } \\
\text { con poca pendiente }\end{array}$ \\
\hline $\begin{array}{l}\text { El } \\
\text { Holandés }\end{array}$ & 21.8228 & -84.7808 & $\begin{array}{l}\text { Terraza } \\
\text { areno-rocosa }\end{array}$ & $\begin{array}{l}\text { Camellones y } \\
\text { canales }\end{array}$ & $\begin{array}{l}\text { Camellones y } \\
\text { canales }\end{array}$ & $\begin{array}{l}\text { Camellones y } \\
\text { canales con poca } \\
\text { pendiente }\end{array}$ \\
\hline $\begin{array}{l}\text { Caleta del } \\
\text { Piojo }\end{array}$ & 21.8233 & -84.8713 & $\begin{array}{l}\text { Terraza } \\
\text { rocosa }\end{array}$ & $\begin{array}{l}\text { Camellones y } \\
\text { canales }\end{array}$ & $\begin{array}{l}\text { Camellones y } \\
\text { canales }\end{array}$ & $\begin{array}{l}\text { Camellones y } \\
\text { canales }\end{array}$ \\
\hline $\begin{array}{l}\text { Caleta } \\
\text { Larga }\end{array}$ & 21.8306 & -84.9264 & $\begin{array}{l}\text { Terraza } \\
\text { rocosa }\end{array}$ & $\begin{array}{l}\text { Borde } \\
\text { superior del } \\
\text { veril }\end{array}$ & $\begin{array}{l}\text { Borde exterior } \\
\text { del veril }\end{array}$ & Arenal* \\
\hline $\begin{array}{l}\text { Faro } \\
\text { Roncali }\end{array}$ & 21.8685 & -84.9585 & $\begin{array}{l}\text { Cabezos } \\
\text { coralinos }\end{array}$ & $\begin{array}{l}\text { Borde } \\
\text { exterior del } \\
\text { veril }\end{array}$ & Arenal* & Arenal* \\
\hline
\end{tabular}

definieron las especies dominantes en vivo relativa, que incluye las especies estos arrecifes.

A la matriz de datos (transformada 95\% del total, se le aplicó un Análisis a raíz cuadrada) de cobertura de coral de Clasificación Numérica Jerárquica 
Aglomerativa, con el fin de conocer la similitud entre las estaciones mediante el coeficiente de Similitud de Bray Curtis (Boesch, 1977). Para conformar los dendogramas se empleó el método de agrupamiento de Promedio entre Grupos (UPGMA) (Boesch, 1977), utilizando el Programa Primer 5.5 (PRIMER-E, 2002). Se sumó el porcentaje de cobertura de coral vivo relativa de las especies consideradas por Hubbard (1986) y Torres \& Morelock (2002) como resistentes a la sedimentación $(S$. siderea, $P$. astreoides, D. strigosa y $M$. cavernosa) como un indicador comparativo de intensidad de ese factor.

La riqueza de especies fue considerada como el número de especies en cada estación, una vez que se verificó que las curvas acumulativas de especies en relación con el número de transectos tendieron a estabilizarse en todos los casos. Estas curvas fueron obtenidas y suavizadas mediante permutación aleatoria (999 permutaciones) del orden de las muestras con el Programa Primer 5.5 (PRIMER-E, 2002). Se determinaron la talla media de corales y los porcentajes promedio de superficie afectada por mortalidad antigua y reciente por estación.

Para conocer la significación de las diferencias entre las estaciones de una misma profundidad, en relación con los promedios de cobertura, talla y mortalidad antigua de corales, se aplicaron análisis de varianza unifactoriales. Se comprobó la normalidad y homogeneidad de varianza de los datos a partir de la relación propuesta por Taylor (1961), según la metodología de Elliot (1971). Se aplicó la transformación de los datos reales cuando fue necesario, según lo establecido por la mencionada Ley de Taylor. Se comprobó que estas transformaciones normalizaron los datos. Para detectar diferencias entre los pares de medias se utilizó la prueba de Student-Newman-Keuls (SNK), con un nivel de significación de 0.05 . Los cálculos se realizaron con la versión 7.0 del Programa Statistica (StatSoft Inc., 2004).

\section{RESULTADOS}

\section{Cobertura}

La cobertura de coral vivo en el área varió entre $5 \%$ y $31 \%$, con un promedio general de 17\% (Fig. 2). En los $5 \mathrm{~m}$ de profundidad, las estaciones ubicadas entre Veral y Caleta Larga no exhibieron desarrollo coralino al igual que en Cuevas de Pedro. La cobertura de coral vivo de la estación Faro Roncali fue significativamente mayor que en las estaciones María la Gorda y La Bajada, entre las cuales la diferencia no fue estadísticamente significativa. Los restantes promedios tuvieron una posición intermedia, sin diferencias significativas.

A 10 m de profundidad no se observó cobertura coralina en Verraco ni en Resguardo a causa de la presencia de una gruesa capa de arena sobre el sustrato. Los mayores valores de cobertura de coral vivo fueron compartidos por varias estaciones sin diferencias significativas entre sí (Faro Roncali, El Holandés, La Bajada, Uvero, María la Gorda y Cuevas de Pedro), mientras que los menores se obtuvieron en Caleta del Piojo, Caleta 

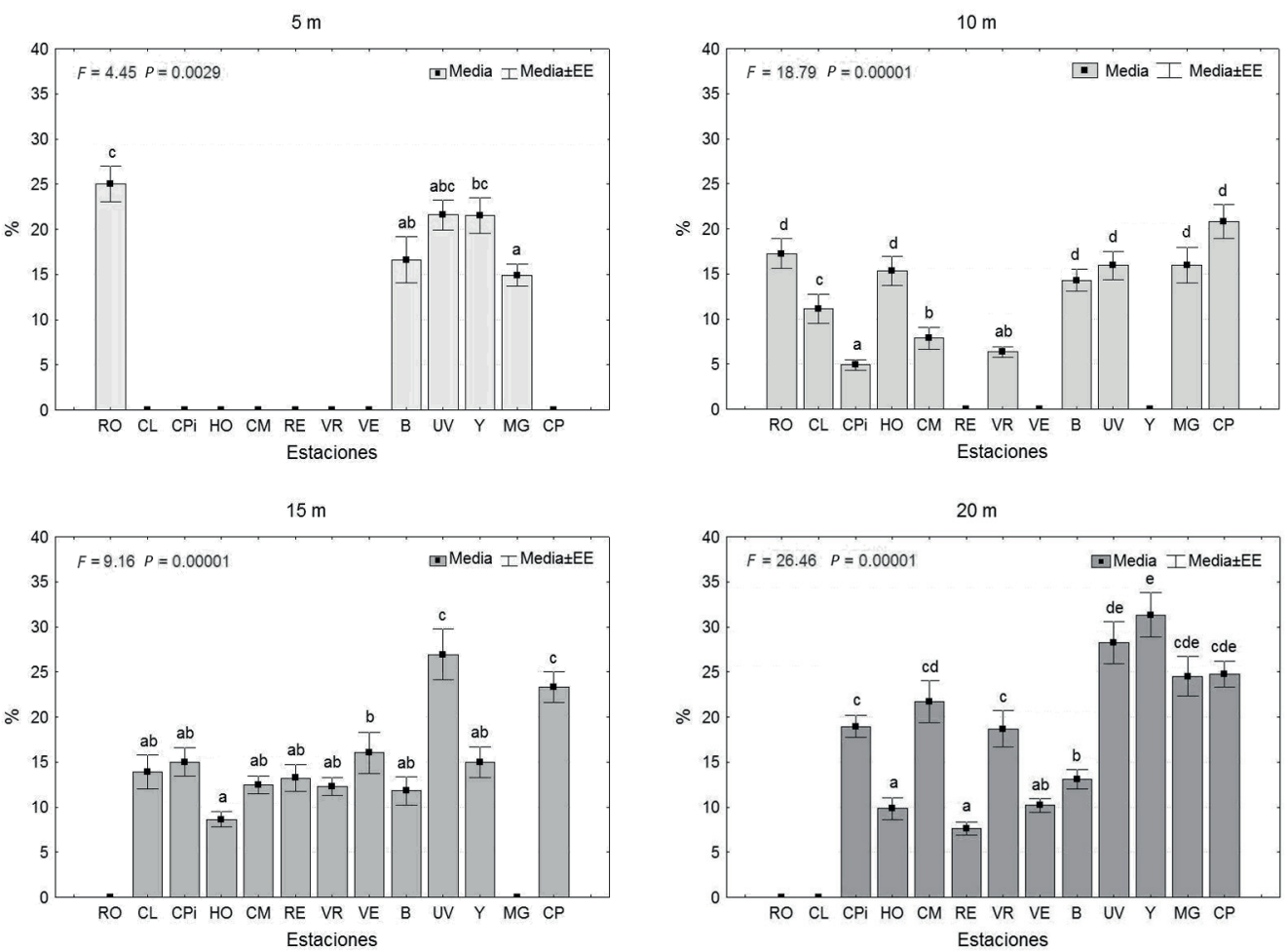

Fig. 2. Variación espacial de la cobertura de coral vivo (resultados del ANOVA de clasificación simple). Las letras muestran el resultado del análisis de SNK. RO: Faro Roncali, CL: Caleta Larga, CPi: Caleta del Piojo, HO: El Holandés, CM: Caleta del Mangle, RE: Resguardo, VR: Verraco, VE: Veral, B: La Bajada, UV: Uvero, Y: Yemayá, MG: María la Gorda, CP: Cuevas de Pedro Fig. 2. Spatial variability of live coral cover (results of one-way ANOVA). The letters show the results of the SNK analysis. RO: Faro Roncali, CL: Caleta Larga, CPi: Caleta del Piojo, HO: El Holandés, CM: Caleta del Mangle, RE: Resguardo, VR: Verraco, VE: Veral, B: La Bajada, UV: Uvero, Y: Yemayá, MG: María la Gorda, CP: Cuevas de Pedro

del Mangle y Verraco, donde solo los dos primeros fueron significativamente diferentes.

En los $15 \mathrm{~m}$ de profundidad, Uvero y Cuevas de Pedro se destacaron significativamente del resto de las estaciones. En María la Gorda, la cobertura de coral vivo fue nula, dada la carencia de sustrato adecuado.

A $20 \mathrm{~m}$ de profundidad, las estaciones Yemayá, Uvero, María la Gorda y Cuevas de Pedro resaltan de manera significativa del resto por su mayor cobertura.

\section{Especies predominantes}

En el área de estudio se identificaron 42 especies de corales pétreos pertenecientes a dos órdenes, 10 familias y 20 géneros. Las especies con mayor cobertura de coral vivo relativa fueron $S$. siderea y Montastraea faveolata, seguidas por Agaricia agaricites y 
P. astreoides. También se destacaron Agaricia lamarcki, M. cavernosa y Stephanocoenia intersepta. Estas siete especies son las más representativas, ya que contribuyeron al $80 \%$ de la cobertura general del área.

Al analizar por profundidades, $S$. siderea dominó como promedio general (Fig. 3) y en todas las estaciones a $5 \mathrm{~m}$ de profundidad (Cuadro 2).

Cuadro 2. Número de especies (S) y especie dominante por estaciones. RO: Faro Roncali, CL: Caleta Larga, CPi: Caleta del Piojo, HO: El Holandés, CM: Caleta del Mangle, RE: Resguardo, VR: Verraco, VE: Veral, B: La Bajada, UV: Uvero, Y: Yemayá, MG: María la Gorda, CP: Cuevas de Pedro, Ssid: S. siderea, Aaga: A. agaricites, Mfav: M. faveolata, Alam: A. lamarcki, Mcav: M. cavernosa

Table 2. Number of species (S) and dominant species by station. RO: Faro Roncali, CL: Caleta Larga, CPi: Caleta del Piojo, HO: El Holandés, CM: Caleta del Mangle, RE: Resguardo, VR: Verraco, VE: Veral, B: La Bajada, UV: Uvero, Y: Yemayá, MG: María la Gorda, CP: Cuevas de Pedro, Ssid: S. siderea, Aaga: A. agaricites, Mfav: M. faveolata, Alam: A. lamarcki, Mcav: M. cavernosa

\begin{tabular}{|c|c|c|c|c|c|c|c|c|c|c|c|c|c|c|}
\hline \multirow{2}{*}{ Prof. } & \multirow{2}{*}{ Índices } & \multicolumn{13}{|c|}{ Estaciones } \\
\hline & & RO & CL & $\mathbf{C P i}$ & HO & $\mathbf{C M}$ & RE & VR & VE & B & UV & $\mathbf{Y}$ & MG & $\mathrm{CP}$ \\
\hline \multirow{2}{*}{$5 \mathrm{~m}$} & $\mathbf{S}$ & 20 & & & & & & & & 14 & 19 & 20 & 18 & \\
\hline & Sp. dom. & Ssid & & & & & & & & Ssid & Ssid & Ssid & Ssid & \\
\hline \multirow{2}{*}{$10 \mathrm{~m}$} & $\mathbf{S}$ & 14 & 15 & 11 & 16 & 13 & & 8 & & 15 & 22 & & 19 & 19 \\
\hline & Sp. dom. & Mcav & Ssid & Ssid & Ssid & Ssid & Ssid & Ssid & Ssid & Ssid & Mfav & & Mfav & Mfav \\
\hline \multirow{2}{*}{$15 \mathrm{~m}$} & $\mathbf{S}$ & & 14 & 15 & 14 & 15 & 16 & 14 & 16 & 17 & 18 & 23 & & 20 \\
\hline & Sp. dom. & Aaga & Mfav & Ssid & Alam & Ssid & Ssid & Ssid & Mfav & Ssid & Mfav & Aaga & & Mfav \\
\hline \multirow{2}{*}{$20 \mathrm{~m}$} & $\mathbf{S}$ & & & 19 & 20 & 17 & 13 & 17 & 16 & 18 & 19 & 19 & 19 & 16 \\
\hline & Sp. dom. & & & Mfav & Ssid & Mfav & Alam & Mfav & Mfav & Mfav & Alam & Mfav & Mfav & Mfav \\
\hline
\end{tabular}

Las especies $A$. agaricites, $M$. annularis, M. faveolata y P. astreoides también ocuparon un lugar importante en la cobertura de coral vivo en los $5 \mathrm{~m}$.

En los $10 \mathrm{~m}, S$. siderea predominó marcadamente sobre las especies restantes (Fig. 3), contribuyendo al 40\% de la cobertura de coral vivo promedio de esa profundidad. Esta especie fue importante por su cobertura de coral vivo en las estaciones comprendidas entre La Bajada y Caleta Larga.
En Uvero, María la Gorda y Cuevas de Pedro (estaciones más orientales) prevaleció $M$. faveolata (Cuadro 2). El tercer lugar en dominancia lo ocupó $A$. agaricites (Fig. 3), por sus importantes contribuciones a la cobertura de coral vivo en Uvero, María la Gorda y Cuevas de Pedro.

En la profundidad de $15 \mathrm{~m}$ también dominó como promedio $S$. sidérea, seguida por M. faveolata, esta última con un valor bastante cercano a la primera, 
$5 \mathbf{m}$

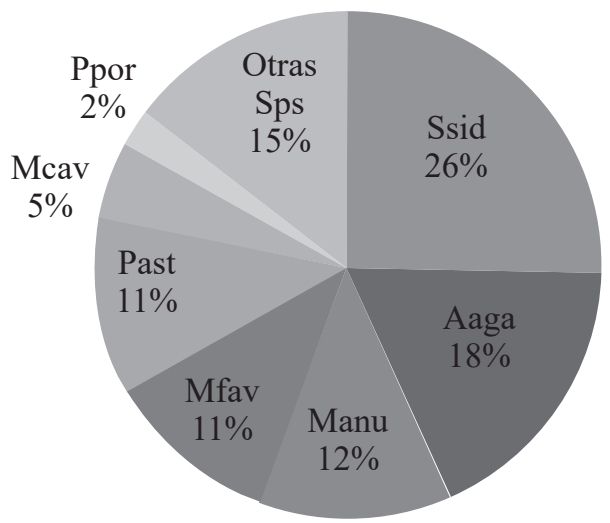

$15 \mathrm{~m}$

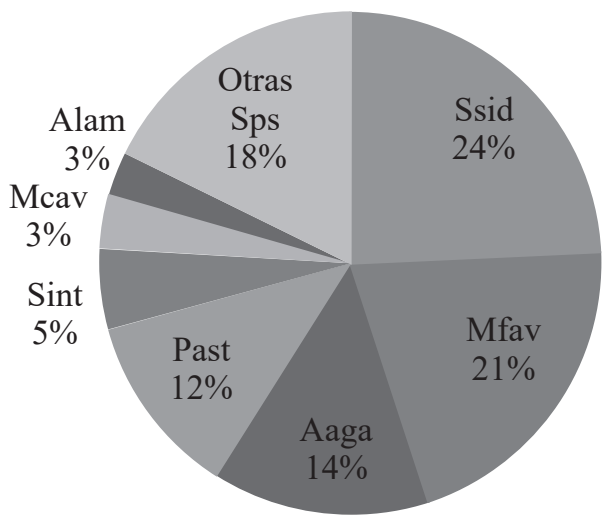

$10 \mathrm{~m}$

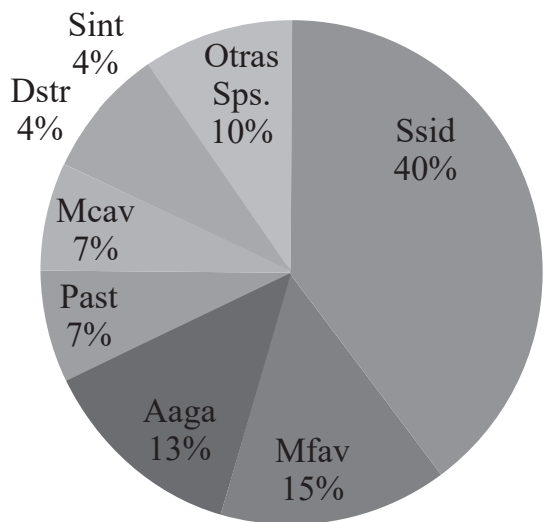

$20 \mathrm{~m}$

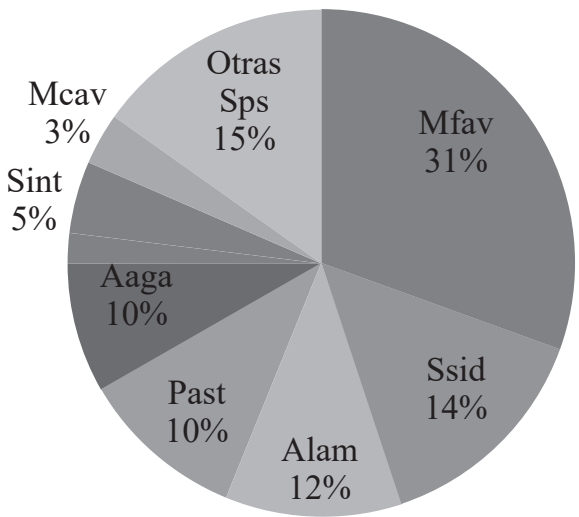

Fig. 3. Dominancia de especies por profundidades, según su aporte al porcentaje de cubrimiento vivo de coral (cubrimiento relativo). Ssid: S. siderea, Aaga: A. agaricites, Mann: Montastraea annularis, Mfav: M. faveolata, Past: P. astreoides, Mcav: M. cavernosa, Ppor: Porites porites, Dstr: Diploria strigosa, Sint: S. intersepta, Alam: A. lamarcki, Otras Spp: Otras especies

Fig. 3. Species dominance by depth, based on the percentage of live coral cover (relative cover). Ssid: S. siderea, Aaga: A. agaricites, Mann: Montastraea annularis, Mfav: M. faveolata, Past: P. astreoides, Mcav: M. cavernosa, Ppor: Porites porites, Dstr: Diploria strigosa, Sint: S. intersepta, Alam: A. lamarcki, Otras Spp: Other species

mientras que en tercer lugar estuvo $A$. agaricites (Fig. 3). En el análisis por estaciones, $S$. siderea predominó en El Holandés, Caleta del Mangle, Resguardo, Verraco y La Bajada; M. faveolata, en Caleta del Piojo, Veral, Uvero y Cuevas de Pedro, mientras que $A$. agaricites, en Caleta Larga y Yemayá (Cuadro 2).
En los 20 m, M. faveolata ocupó el primer lugar en su aporte a la cobertura general, dominando en todas las estaciones, excepto en Resguardo, Uvero y El Holandés. En las dos primeras predominó A. lamarcki, mientras que en la última, S. siderea (Fig. 3).

Similitud de comunidades de corales pétreos entre estaciones 
En los $5 \mathrm{~m}$ hubo una marcada similitud (Fig. 4) entre todas las estaciones (70\%), aunque ésta se acentuó entre Uvero y Yemayá (estaciones contiguas).

En los $10 \mathrm{~m}$ se obtuvieron tres grupos: uno con solo la estación Faro Roncali, otro con La Bajada, Uvero, María la Gorda y Cuevas de Pedro (geográficamente cercanas entre sí) y un tercero con el resto de las estaciones ubicadas geográficamente entre Verraco y Caleta Larga (Fig. 4).

En las profundidades de 15 y $20 \mathrm{~m}$ se presentó una tendencia de agrupación espacial de las estaciones, aunque algo
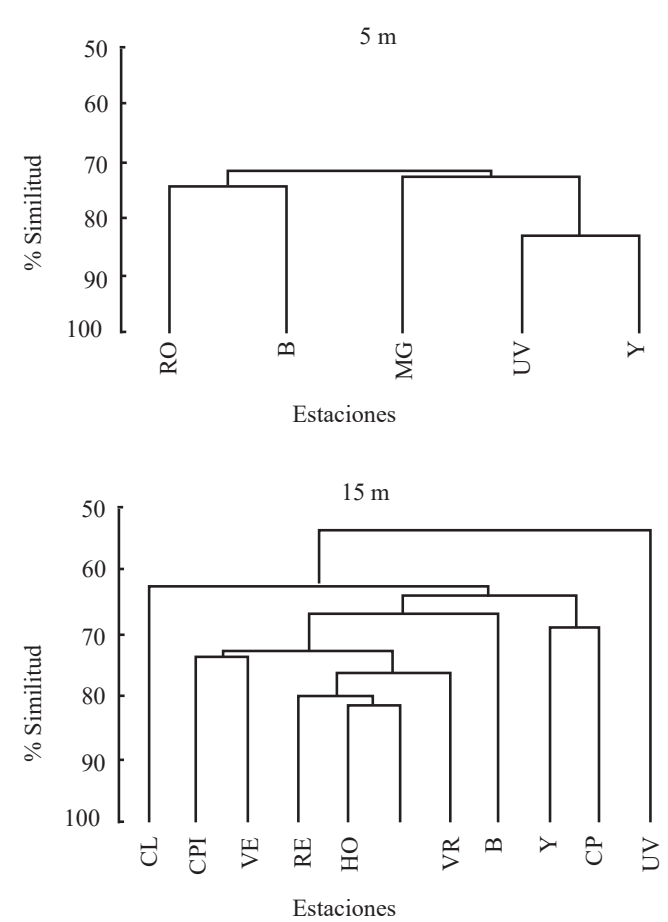

menor. En los $15 \mathrm{~m}$ se formaron cinco grupos: uno con las estaciones ubicadas entre Veral y Caleta del Piojo (estaciones consecutivas), otro constituido por las estaciones Yemayá y Cuevas de Pedro, cercanas geográficamente, y por último tres grupos formados por una estación cada uno: La Bajada, Uvero y Caleta Larga (Fig. 4). En los $20 \mathrm{~m}$ se separó marcadamente El Holandés de otro grupo que incluye al resto de las estaciones, aunque también se apartan ligeramente Uvero, Yemayá y María la Gorda, contiguas entre sí y ubicadas en la zona oriental del área (Fig. 4).
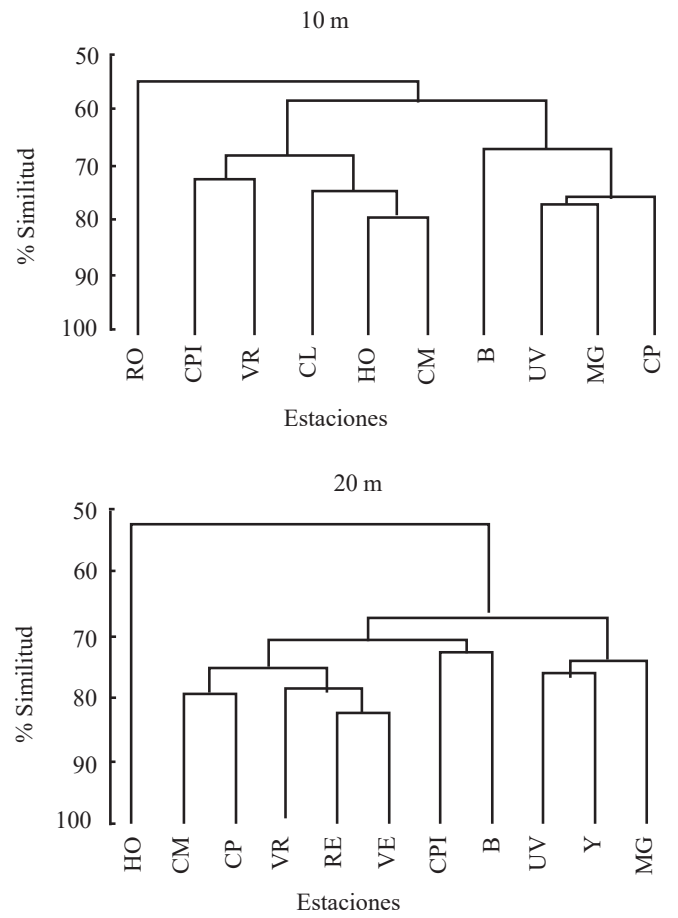

Fig. 4. Dendrogramas que muestran el grado de similitud entre las estaciones según el porcentaje de cubrimiento vivo de las especies. RO: Faro Roncali, CL: Caleta Larga, CPi: Caleta del Piojo, HO: El Holandés, CM: Caleta del Mangle, RE: Resguardo, VR: Verraco, VE: Veral, B: La Bajada, UV: Uvero, Y: Yemayá, MG: María la Gorda, CP: Cuevas de Pedro

Fig. 4. Dendrograms showing the similitude level between the studied sites, based on the percentage of live coral cover. RO: Faro Roncali, CL: Caleta Larga, CPi: Caleta del Piojo, HO: El Holandés, CM: Caleta del Mangle, RE: Resguardo, VR: Verraco, VE: Veral, B: La Bajada, UV: Uvero, Y: Yemayá, MG: María la Gorda, CP: Cuevas de Pedro 


\section{Predominio de especies resistentes a la sedimentación}

El porcentaje de cobertura de especies resistentes a la sedimentación mostró diferencias dependiendo de la profundidad analizada (Fig. 5). En los $5 \mathrm{~m}$, el aporte de este conjunto de especies a la cobertura fue parecido entre las estaciones. En las restantes profundidades se observaron diferencias espaciales en este indicador, siendo mayor la cobertura de este grupo de especies hacia el oeste, sobre todo entre 15 y $20 \mathrm{~m}$.

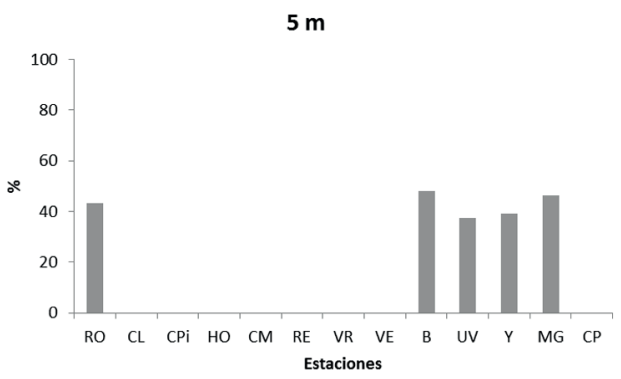

$15 \mathrm{~m}$

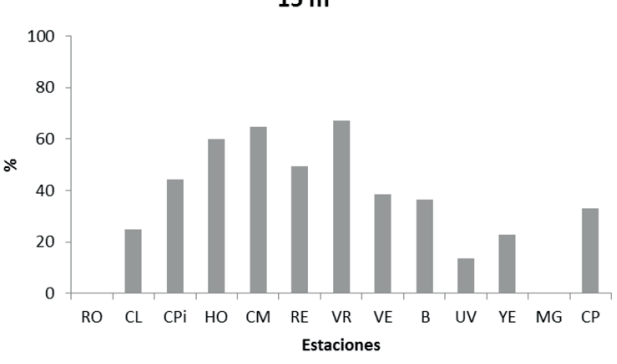

\section{Riqueza de especies}

En las estaciones de $5 \mathrm{~m}$, la riqueza de especies varió entre 14 y 20 especies, con los mayores valores en Yemayá y Faro Roncali. En los 10 m osciló entre 8 y 22 especies, siendo mayor en las estaciones Uvero, María la Gorda y Cuevas de Pedro, situadas al este de la Bahía de Corrientes. En la profundidad de $15 \mathrm{~m}$, el número de especies varió entre 14 y 23, siendo mayor en Yemayá, que es una de las estaciones orientales.

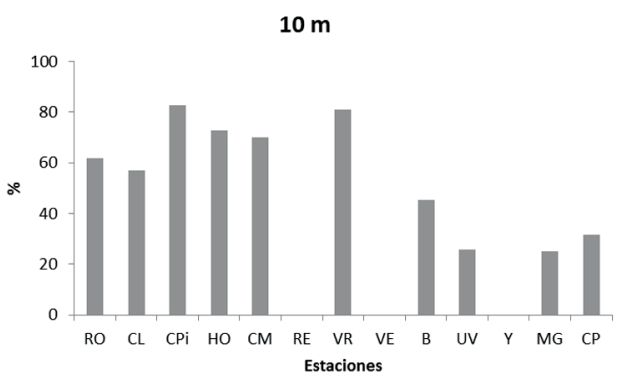

Fig. 5. Variación espacial del cubrimiento relativo de especies resistentes a la sedimentación (S. siderea, P. astreoides, M. cavernosa y D. strigosa). RO: Faro Roncali, CL: Caleta Larga, CPi: Caleta del Piojo, HO: El Holandés, CM: Caleta del Mangle, RE: Resguardo, VR: Verraco, VE: Veral, B: La Bajada, UV: Uvero, Y: Yemayá, MG: María la Gorda, CP: Cuevas de Pedro

Fig. 5. Spatial variability of relative coral cover of species resistant to sedimentation ( $S$. siderea, P. astreoides, M. cavernosa and D. strigosa). RO: Faro Roncali, CL: Caleta Larga, CPi: Caleta del Piojo, HO: El Holandés, CM: Caleta del Mangle, RE: Resguardo, VR: Verraco, VE: Veral, B: La Bajada, UV: Uvero, Y: Yemayá, MG: María la Gorda, CP: Cuevas de Pedro 
En las estaciones de $20 \mathrm{~m}$, la riqueza de especies varió poco entre estaciones, entre 16 y 20 especies, excepto en Resguardo con solo 13 (Cuadro 2).

\section{Diámetro máximo de los corales pétreos}

Los diámetros máximos exhibieron valores promedio superiores (Fig. 6) $\mathrm{y}$ una tendencia a un mayor número de colonias grandes en las estaciones más orientales. En los $5 \mathrm{~m}$, Yemayá mostró el mayor promedio (Fig. 6), diferenciándose significativamente de
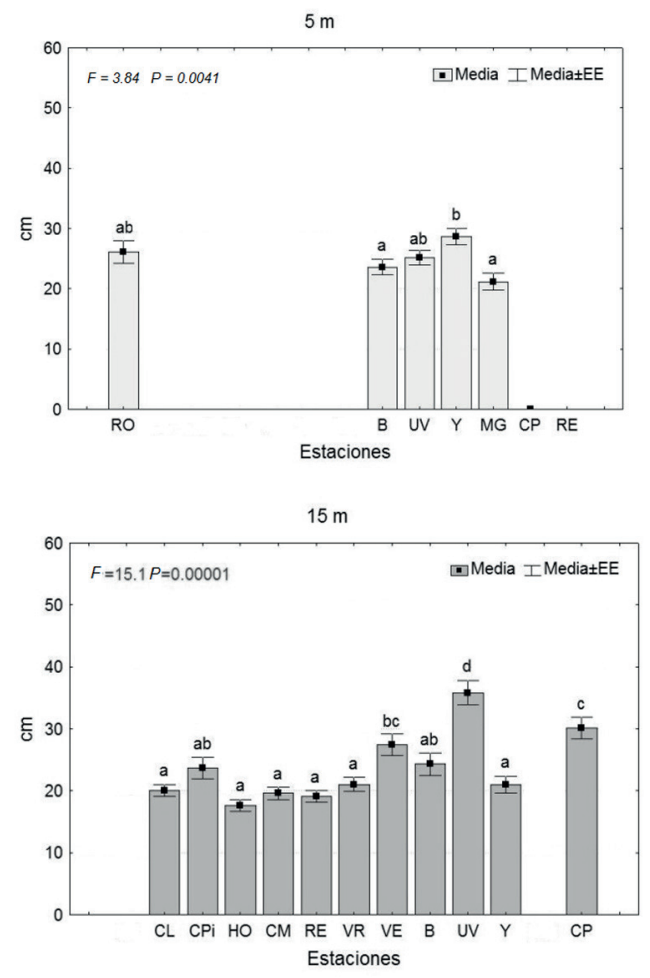

La Bajada y María la Gorda.

En la profundidad de $10 \mathrm{~m}$, Cuevas de Pedro presentó la mayor talla promedio de las colonias (Fig. 6), difiriendo significativamente de las restantes estaciones, excepto de Uvero y El Holandés. En esta profundidad, solamente se observaron colonias mayores de $100 \mathrm{~cm}$ en María la Gorda, Cuevas de Pedro, Uvero y El Holandés.

En los $15 \mathrm{~m}$, la talla promedio de Uvero difirió significativamente de las restantes estaciones (Fig. 6). En los
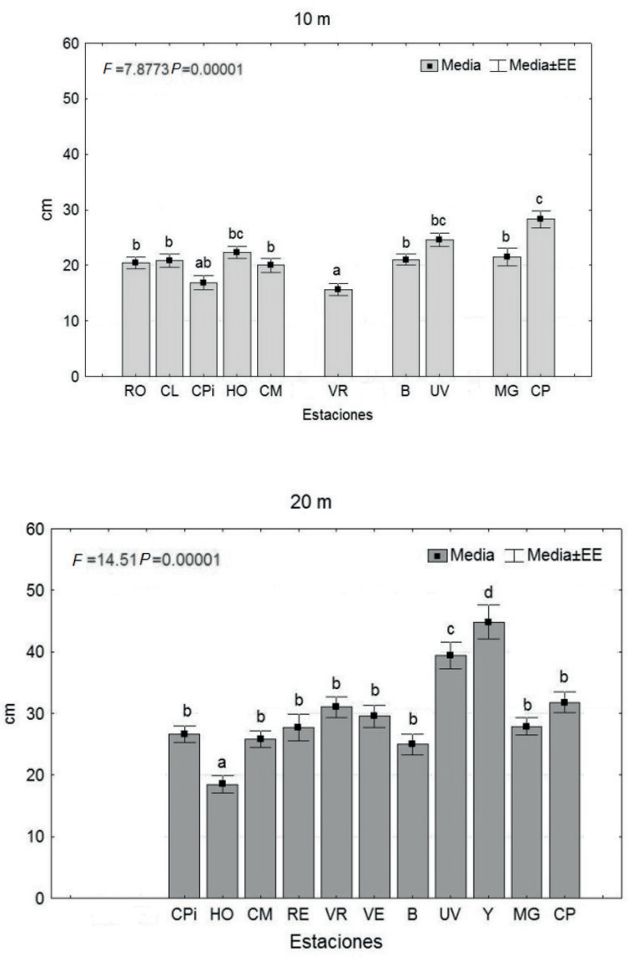

Fig. 6. Variación espacial del diámetro máximo de las colonias (resultados del ANOVA de clasificación simple). Las letras muestran los resultados del análisis de SNK. RO: Faro Roncali, CL: Caleta Larga, CPi: Caleta del Piojo, HO: El Holandés, CM: Caleta del Mangle, RE: Resguardo, VR: Verraco, VE: Veral, B: La Bajada, UV: Uvero, Y: Yemayá, MG: María la Gorda, CP: Cuevas de Pedro Fig. 6. Spatial variability of the maximum diameter of the colonies (results of one-way ANOVA). The letters show the results of the SNK analysis. RO: Faro Roncali, CL: Caleta Larga, CPi: Caleta del Piojo, HO: El Holandés, CM: Caleta del Mangle, RE: Resguardo, VR: Verraco, VE: Veral, B: La Bajada, UV: Uvero, Y: Yemayá, MG: María la Gorda, CP: Cuevas de Pedro 
20 m, la estación Yemayá presentó el mayor promedio de talla, seguida por Uvero (Fig. 6). Ambas difirieron significativamente de las restantes y a su vez entre sí.

\section{Mortalidad antigua}

La mortalidad antigua promedio varió entre el $2 \%$ y $18 \%$ en el conjunto de las estaciones, registrándose el mayor porcentaje en Yemayá a $5 \mathrm{~m}$. En la profundidad de $10 \mathrm{~m}$, la mortalidad antigua fue de menos de $10 \%$, excepto en las estaciones orientales de Uvero y Cuevas de Pedro. En los $15 \mathrm{~m}$ de profundidad, los mayores porcentajes se registraron en Veral y La Bajada, mientras que de las estaciones más profundas $(20 \mathrm{~m})$, Verraco mostró el mayor porcentaje.

\section{Mortalidad reciente, enfermedades infecciosas y blanqueamiento de corales}

En las estaciones en que se detectó mortalidad reciente, esta fue muy baja (menor de 1\%). La incidencia de enfermedades infecciosas y de blanqueamiento varió entre nula y $1 \%$ de corales afectados.

\section{DISCUSIÓN}

Las variaciones espaciales de la estructura de las comunidades de corales del Parque Nacional Guanahacabibes, expresadas en la cobertura de coral vivo, el diámetro máximo promedio y la riqueza de especies, sugieren la presencia de dos amplias regiones sometidas a regímenes generadores de oleaje y sedimentación diferentes, los cuales se reflejan en su estado de salud. Esto se evidenció en la agrupación de las estaciones en los dendogramas (Fig. 4). Los arrecifes con mejores indicadores de condición se ubicaron en la zona más resguardada, al este del área. Esto se concuerda con lo planteado por Storlazzi et al. (2002), quienes establecen que los arrecifes más desarrollados, con mayor cobertura de coral y mayor diversidad, aparecen en regímenes de baja energía. Ratificando lo anterior, los resultados del presente trabajo revelan que en la zona más expuesta a los vientos (entre Veral y Faro Roncali) se observaron los menores valores de cobertura de coral vivo y de riqueza de especies, así como una mayor cobertura relativa del conjunto de las especies resistentes a los efectos de los sedimentos, sobre todo en los 10 y $15 \mathrm{~m}$, donde estos se acumulan más.

Aparentemente, debido a la disminución del efecto determinístico de factores océano-atmosféricos generadores de oleaje y de dinámica de los sedimentos del fondo, el patrón de variación espacial que los refleja se manifiesta en menor medida a mayor profundidad $(20 \mathrm{~m})$. A esta profundidad, el porcentaje de especies resistentes a la sedimentación fue más bajo y a la vez parecido en todas las estaciones (excepto en El Holandés). Según Alcolado (1999), en las estaciones más profundas se produce una mejoría en las condiciones ambientales dada por la disminución del efecto de la sedimentación y la abrasión (menor frecuencia e intensidad de la suspensión 
de sedimentos por el oleaje, pendientes mayores e irregularidad del fondo). El hecho de que el efecto de los sedimentos no sea menor en El Holandés a los $20 \mathrm{~m}$, puede ser consecuencia de que esta estación sea la más afectada por los vientos durante la mayor parte del año y con un gran fetch en la generación del oleaje. Estas características, unidas al más frecuente paso de huracanes por la zona, deben producir de manera más frecuente la desestabilización de los sedimentos hasta en las zonas algo más profundas y pueden haber permitido el desarrollo de comunidades de corales más adaptadas, explicando la alta abundancia relativa de las mencionadas especies resistentes a la sedimentación y el oleaje.

La existencia de los mayores porcentajes de mortalidad antigua en algunas de las estaciones más resguardadas, fundamentalmente en 5 y $10 \mathrm{~m}$, puede ser consecuencia de que en estas estaciones se encuentran las colonias de mayor talla y con mayor mortalidad parcial acumulada debido a su antigüedad. De hecho, Kramer (2003) observó una correlación positiva entre el diámetro y el porcentaje de mortalidad antigua de los corales.

Según el sistema de escalas de un conjunto de indicadores biológicos AGRRA propuesto por Alcolado \& Durán (2011) para evaluar de forma integral la salud o condición de los arrecifes coralinos (basados en datos del Gran Caribe obtenidos mediante el protocolo de indicadores de AGRRA), los resultados del presente trabajo califican la condición de los arrecifes del Parque Nacional Guanahacabibes entre buena y regular. Esto se refleja en los valores obtenidos para los principales indicadores de la condición de los arrecifes, que, como ya se vio, fueron más favorables en la zona resguardada ubicada al este del Parque Nacional. En primer lugar, lo sugiere el patrón espacial de la riqueza de especies de corales observado. Este es uno de los indicadores que contribuye, junto con otros, a la inferencia del grado de salud integral del arrecife, exceptuando las crestas arrecifales saludables dominadas por acropóridos $\mathrm{y}$, sin embargo, con poca riqueza de especies. Se infiere que un arrecife está en buenas condiciones cuando hay más de 16 especies. Los valores de riqueza de especies se mantuvieron en general por encima de esta cifra en las estaciones orientales más resguardadas, y se mostraron mayormente entre 11 y 16 en las estaciones expuestas, las que se pueden catalogar con condición regular para este indicador biológico (Alcolado \& Durán, 2011).

Otros indicadores que reflejan la existencia de dos regiones diferenciadas en los arrecifes del PNG, en cuanto al nivel de exposición a los vientos, son los porcentajes de cobertura de coral vivo detectados, los cuales sobrepasaron el $20 \%$ del sustrato en varias de las estaciones ubicadas al este del parque y por regla general se mantuvieron por encima del $10 \%$ en las estaciones más occidentales. Alcolado \& Durán (2011) consideran que una cobertura por encima del $20 \%$ es típico de arrecifes saludables, mientras que una cobertura 
de coral vivo entre 10 y $20 \%$ refleja un regular estado de salud. Este indicador es considerado uno de los más confiables para determinar el estado de salud del arrecife, por su fácil estimación y respuesta rápida a las presiones ambientales negativas en los arrecifes (McField \& Kramer, 2007).

La composición por especies del área de estudio refleja alta dominancia de aquellas consideradas como principales formadoras de arrecifes, entre ellas las del complejo M. annularis, así como $M$. cavernosa, $S$. siderea y $A$. agaricites. Muchas de estas especies han sufrido alta mortalidad en los arrecifes del Caribe, llegando a desaparecer en algunos de ellos (Creary et al. 2008; García-Salgado et al. 2008). En los arrecifes de la zona más resguardada del parque se encontraron con frecuencia ejemplares de hasta 1 $\mathrm{m}$ de diámetro, lo que indica que aún persisten colonias con resistencia a las alteraciones climáticas globales. Por otra parte, el predominio de $S$. siderea, $P$. astreoides y en menor medida de $M$. cavernosa, en la mayoría de las estaciones de muestreo, probablemente indica que los efectos de la sedimentación están presentes en todos los arrecifes del Parque Nacional, los cuales con frecuencia se ven sometidos al efecto de huracanes. Estas especies son consideradas como resistentes a la acción de sedimentos y del oleaje, y son capaces de soportar daño mecánico y fuerte iluminación (Hubbard, 1986; Torres \& Morelock, 2002).

La codominancia de M. faveolata, en cobertura relativajunto con $S$. siderea, y su mayor predominio en casi todas las estaciones de $20 \mathrm{~m}$ y en la mayoría de las estaciones de 10 y $15 \mathrm{~m}$ situadas al este del área, permite hacer inferencias sobre la resistencia de esta especie a la sedimentación. Esta especie no se considera tan resistente como $S$. siderea, pero González-Ferrer (2004) le atribuye cierta tolerancia a la sedimentación y al estrés hidrometeorológico. $M$. faveolata se considera como indicador de la antigüedad y estabilidad de los arrecifes dada su alta susceptibilidad a enfermedades (Kramer, 2003).

La especie $A$. agaricites no es considerada resistente a la sedimentación y es muy vulnerable a lesiones en su tejido e invasión de organismos excavadores (Torres \& Morelock, 2002). Sin embargo, su tasa de reproducción es alta y suelen ser abundantes las pequeñas colonias (Bak \& Stewardvan Es, 1980), sobre todo en bajas profundidades, lo que puede explicar por qué ocupó el tercer lugar en predominio general de cobertura relativa.

Según Alcolado \& Durán (2011), la mortalidad reciente también se emplea para calcular el índice de salud arrecifal, y establecen que menos del $2 \%$ de mortalidad reciente puede ser considerado un valor favorable para ese indicador específico. Esto puede deberse a que las causas de estrés que más generan mortalidad reciente ocurrieron más de 1 año antes del muestreo. En este sentido, los últimos huracanes de gran intensidad sucedieron en la zona en el 2004 y 2005, y el evento de blanqueamiento más intenso antes del estudio fue en el 2005 (Wilkinson \& 
Souter, 2008). Además, a partir de los valores muy bajos de mortalidad reciente, se infiere que durante el último año antes de los muestreos no existieron afectaciones importantes por enfermedades microbianas. Esto apoya el criterio de varios autores sobre una aparente tendencia a la disminución de su frecuencia en el Gran Caribe (Miller et al. 2003; Kim \& Harvell, 2004; Reshef et al. 2006; Lesser et al. 2007).

\section{BIBLIOGRAFÍA}

AGRRA. (2000). The AGRRA Rapid Assessment Protocol. Atlantic and Gulf Rapid Reef Assessment Methodology. Recuperado en abril 17, 2009, disponible en http://www.agrra.org/index. html

Alcolado, P. (1999). Comunidades de esponjas de los arrecifes del archipiélago Sabana-Camagüey. Bol. Invest. Mar. Cost., 28, 95-124.

Alcolado, P. M., Martínez-Daranas, B., Menéndez-Macia, G., del Valle, R., Hernández, M. \& García, T. (2003). Rapid assessment of coral communities of María la Gorda, southeast Ensenada de Corrientes, Cuba (Part 1: stony corals and algae). En J. C. Lang (Ed.), Status of Coral Reefs in the Western Atlantic. Atoll. Res. Bull., 496, 268-277.

Alcolado, P. \& Durán, A. (2011). Sistema de escalas para la clasificación y puntaje de condición del bentos e ictiofauna de arrecifes coralinos de Cuba y del Gran Caribe. Serie Oceanológica, 8, 25-29.

Bak, R. P. M. \& Steward-van Es, Y. (1980). Regeneration of superficial damage in the scleractinian corals
Agaricia agaricites F. purpurea and Porites astreoides. Bull. Mar. Sci., 30(4), 883-887.

Boesch, D. F. (1977). Application of numerical classification in ecological investigations of water pollution. Virginia Ecol. Res. Ser., 77, 1-126.

Caballero, H., González, S., Cobián, D., Álvarez, S. \& Alcolado, P. (2007). Evaluación AGRRA del bentos en diez sitios de buceo de "María la Gorda", Bahía de Corrientes, Cuba. Rev. Invest. Mar., 28(2), 131-138.

Creary, M., Alcolado, P., Coelho, V., Crabbe, J., Green, S., Geraldes, F., Henry, A., Hibbert, M., Jones, R., JonesSmith, L., Manfrino, C., Manuel, S., McCoy, C. \& Wiener, J. (2008). Status of Coral Reefs in the Northern Caribbean and Western Atlantic GCRMN Node in 2008. En C. Wilkinson (Ed.), Status of the Coral Reefs of the World: 2008 (pp. 239-252). Global Coral Reef Monitoring Network and Reef and Rainforest Research Center. Townsville, Australia: Australian Institute of Marine Science Press.

Cuba. (2001a). Resolución Legal No. $40 / 2003$ Ministerio de la Industria Pesquera, de 2 de julio, por la que se declara como Zona bajo régimen especial de uso y protección las aguas marítimas comprendidas a partir del litoral de la península de Guanahacabibes hasta 1 milla mar afuera, desde Cayos de La Leña hasta Jaimanitas. Gaceta Oficial de la República de Cuba. 033, 12-15.

Cuba. (2001b). Acuerdo Legal 4262 /2001, de 14 de diciembre, por el que se declaran 32 áreas del territorio nacional como áreas protegidas. Gaceta Oficial de la República de Cuba. 091, 21-39. 
Díaz-Pulido, G., Sánchez, J. A., Zea, S., Díaz, J. M. \& Garzón, J. (2004). Esquemas de distribución espacial en la comunidad bentónica de arrecifes coralinos continentales y oceánicos del Caribe Colombiano. Rev. Acad. Colomb. Cienc., 28(108), 337-347.

Elliot, J. M. (1971). Some methods for the statistical analysis of samples of benthic invertebrates. Sci. Publ., 25, 144-172.

García-Salgado, M., Nava-Martínez, G., Bood, N., Mcfield, M., Molina-Ramírez, A., Yañez-Rivera, B., Jacobs, N., Shank, B., Vázquez, M., Majil, I., Cubas, A., Domínguez-Calderón, J. J. \& Arrivillaga, A. (2008). Status of Coral Reefs in the Mesoamerican Region. En C. Wilkinson (Ed.), Status of the Coral Reefs of the World: 2008 (pp. 253264). Global Coral Reef Monitoring Network and Reef and Rainforest Research Center. Townsville, Australia: Australian Institute of Marine Science Press.

González-Ferrer, S. (2004). Catálogo de los corales hermatípicos de aguas cubanas. En S. González-Ferrer (Ed.), Corales pétreos. Jardines sumergidos de Cuba (pp. 79-189). Madrid, España: Editorial Academia.

González-Ferrer, S., Caballero, H., Alcolado, P. M., Jiménez, A., Martín, F. \& Cobián, D. (2007). Diversidad de corales pétreos en once sitios de buceo recreativo de "María la Gorda", Cuba. Rev. Inves. Mar., 28(2), 121-130.

Hoegh-Guldberg, O. (2004). Coral reefs in a century of rapid environmental change. Symbiosis, 37, 1-31.

Hubbard, D. K. (1986). Sedimentation as a control of reef development: St. Croix, U.S.V.I. Coral Reefs, 5(3), 117-125.

Iturralde-Vinent, M. (2004). Tipos de rocas de Cuba. Recuperado en enero 12,
2009, disponible en http://www.redciencia.cu/cdorigen/arca/trocas.htm

Kim, K. \& Harvell, C. D. (2004). The rise and fall of a six-year coral-fungal epizootic. Am. Nat., 164, S52-S63.

Kramer, P. (2003). Synthesis of coral reef health indicators for the western Atlantic: Results of the AGRRA Program (1997-2000). En J. C. Lang (Ed.), Status of Coral Reefs in the Western Atlantic: Results of Initial Surveys, Atlantic and Gulf Rapid Reef Assessment (AGRRA) Program (pp. 1-57). Washington, EE.UU.: Atoll. Res. Bull., 496.

Lesser, M. P., Bythell, J. C., Gates, R. D., Johnstone, R. W. \& Hoegh-Guldberg, O. (2007). Are infectious diseases rea1ly killing corals? Alternative interpretations of the experimental and ecological data. J. Exp. Mar. Biol. Ecol., 346(1), 36-44.

McField, M. \& Kramer, P. (2007). Healthy Reefs for Healthy People: A guide to Indicators of Reef Health in the Mesoamerican Reef Region. Miami, Fl., EE.UU.: The Smithsonian Institution.

Miller, J., Rogers, C. \& Waara, R. (2003). Monitoring the coral disease, plague type II, on coral reefs in St. John, U.S. Virgin Islands. Rev. Biol. Trop., 51(4), 47-55.

PRIMER-E. (2002). PRIMER 5.5 Plymouth Routines in Multivariate Ecological Research. Plymouth, United Kingdom: PRIMER-E Ltd.

Reshef, L., Koren, O., Loya, Y., ZilberRosenberg, I. \& Rosenberg, E. (2006). The coral probiotic hypothesis. Environ. Microbiol., 8(12), 2068-2073.

Rodríguez, M., González, C. \& Valdés, J. (1984). Cronología de los frentes frios que han afectado a Cuba desde la temporada de 1916-1917 hasta la temporada de 1982-1983. La Habana, Cuba: 
Reporte de Investigación, INSMET, ACC.

Rodríguez-Ramírez, M. E. \& Ballester, M. (1991). Cronología de los Sures que han afectado a la mitad occidental de Cuba (Provincias de Pinar del Río, La Habana, Matanzas e Isla de la Juventud) desde la temporada invernal de 1916-1917 hasta la temporada 1990-1991. La Habana, Cuba: Reporte de Investigación, INSMET, ACC.

Sánchez-Díaz, A. \& Victorero-Hernández, A. (2009). Condiciones climatológicas de la Península de Guanahacabibes (Informe Núm. 1) Pinar del Río. Centro Meteorológico Provincial, INSMET. Pinar del Río, Cuba: CITMA.

StatSoft, Inc. (2004). STATISTICA (Data analysis software system), version 7 . www.statsoft.com. Tulsa, OK.: Statoft, Inc. Steneck, R. S., Lang, J. C., Kramer, P. A. \& Ginsburg, R. N. (1997). AGRRA, Rapid Assessment Protocol. Recuperado en marzo 15, 2007, disponible en http://www.agrra.org/index.html

Storlazzi, C. D., Field, M. E., Dykes, J. D., Jokiel, P. L. \& Brown, E. (2002, septiembre). Wave Control on Reef
Morphology and Coral Distribution: Molokai, Hawaii. Ponencia presentada en la Conferencia WAVES 2001, San Francisco, California.

Taylor, L. R. (1961). Aggregation, variance and the mean. Nature, 189(4766), $732-735$.

Töpfer, K. (2003). Conventions and Coral Reefs. UNEP Coral Reef UnitWWF Coral Reefs Advocacy Initiative. Gland, Switzerland: UNEP Press.

Torres, J. L. \& Morelock, J. N. (2002). Effect of Terrigenous Sediment Influx on Coral Cover and Linear. Caribb. J. Sci., 38(3), 222-229.

Wilkinson, C. \& Souter, D. (2008). Status of Caribbean Coral Reefs After Bleaching and Hurricanes in 2005. Townsville, Australia: Global Coral Reef Monitoring Network, and Reef and Rainforest Research Centre.

Zvuloni, A., van Woesik, R. \& Loya, Y. (2010). Diversity Partitioning of Stony Corals Across Multiple Spatial Scales Around Zanzibar Island, Tanzania. PLoS ONE, 5(3): 1-8. e9941. doi:10.1371/journal. pone. 0009941 